\title{
ON THE LONGEST RNG EDGE OF WIRELESS AD HOC NETWORKS
}

\author{
PENG-JUN WAN* ${ }^{*}$ and LIXIN WANG ${ }^{\dagger}$ \\ Department of Computer Science \\ Illinois Institute of Technology \\ *wan@cs.iit.edu \\ ${ }^{\dagger}$ wanglix@iit.edu \\ FRANCES YAO \\ Department of Computer Science \\ City University of Hong Kong \\ csfyao@cityu.edu.hk \\ CHIH-WEI YI \\ Department of Computer Science \\ National Chiao Tung University \\ yi@cs.nctu.edu.tw
}

Accepted 21 January 2009

\begin{abstract}
Relative neighborhood graph (RNG) has been widely used in topology control and geographic routing in wireless ad hoc networks. Its maximum edge length is the minimum requirement on the maximum transmission radius by those applications of RNG. In this paper, we derive the precise asymptotic probability distribution of the maximum edge length of the RNG on a Poisson point process over a unit-area disk. Since the maximum RNG edge length is a lower bound on the critical transmission radius for greedy forward routing, our result also leads to an improved asymptotic almost sure lower bound on the critical transmission radius for greedy forward routing.
\end{abstract}

Keywords: Relative neighborhood graph; Poisson point process; asymptotic probability distribution.

Mathematics Subject Classification 2000:

\section{Introduction}

Relative neighborhood graph (RNG) [15] of a finite planar set is a bounded-degree planar graph containing the Euclidean minimum spanning tree as a subgraph. Due to its simple construction and maintenance, RNG has found many applications in localized topology control (e.g. $[6,8,9]$ ) and geographic routing (e.g. $[1,7,13])$ in wireless ad hoc networks. All these applications require the maximum transmission 
radius of the networking nodes be no shorter than the longest edge in the RNG. While the maximum edge length in the RNG can be easily computed in polynomial time, little is known about its random behavior when the underlying vertex set is a random point set. In this paper, we derive the precise asymptotic distribution of the maximum edge length in the RNG of a Poisson point process over a unit-area disk with density $n$, which is denoted by $\mathcal{P}_{n}$.

Denote the maximum edge length of a geometric graph $G$ by $\lambda(G)$, and the RNG of a finite planar set $V$ by $R N G(V)$. Denote by $\sigma$ the geometric constant $\frac{2 \pi}{3}-\frac{\sqrt{3}}{2}$ (whose geometric meaning will be explained in the later section). Fix a real parameter $c$ and let

$$
\begin{aligned}
r_{n} & =\sqrt{\frac{\ln n+c}{\sigma n},} \\
\mu & =\frac{\pi}{2 \sigma} e^{-c} .
\end{aligned}
$$

The main result of this paper is stated in the following theorem.

Theorem 1.1. $\lim _{n \rightarrow \infty} \operatorname{Pr}\left[\lambda\left(R N G\left(\mathcal{P}_{n}\right)\right) \leq r_{n}\right]=e^{-\mu}$.

It is interesting to compare the maximum edge length of the RNG with the maximum edge length of the (Euclidean) minimum spanning tree (MST), which is also known the critical transmission radius for connectivity [5], and the maximum edge length of the Gabriel graph (GG) [4], which also has many applications in wireless ad hoc networks. Let $M S T(V)$ and $G G(V)$ denote the MST and the GG of finite planar set $V$. It is well-known that for any finite planar set,

$$
M S T(V) \subseteq R N G(V) \subseteq G G(V) .
$$

Thus,

$$
\lambda(M S T(V)) \leq \lambda(R N G(V)) \leq \lambda(G G(V)) .
$$

The asymptotic distributions of $\lambda\left(M S T\left(\mathcal{P}_{n}\right)\right)$ and $\lambda\left(G G\left(\mathcal{P}_{n}\right)\right)$ were derived in [11] (based on an earlier result [2]) and in [16] respectfully. Specifically, for any constant $c$,

$$
\begin{aligned}
& \lim _{n \rightarrow \infty} \operatorname{Pr}\left[\lambda\left(M S T\left(\mathcal{P}_{n}\right)\right) \leq \sqrt{\frac{\ln n+c}{\pi n}}\right]=e^{-e^{-c}}, \\
& \lim _{n \rightarrow \infty} \operatorname{Pr}\left[\lambda\left(G G\left(\mathcal{P}_{n}\right)\right) \leq 2 \sqrt{\frac{\ln n+c}{\pi n}}\right]=e^{-2 e^{-c}} .
\end{aligned}
$$

So roughly speaking, the maximum edge length of the RNG (respectfully, GG) of a Poisson point process is asymptotically about 1.6 times (respectfully, twice) its critical transmission radius for connectivity.

Another parameter closely related to the maximum edge length of the RNG is the critical transmission radius for greedy forward routing $[3,14]$. In greedy forward routing, each node discards a packet if none of its neighbors is closer to the 
destination of the packet than itself, or otherwise forwards the packet to the neighbor closest to the destination of the packet. The critical transmission radius of a planar node set $V$ for greedy forward routing, denoted by $\phi(V)$, is the smallest transmission radius by $V$ which ensures successful delivery of any packets from any source node in $V$ to any destination node in $V$. Clearly, $\lambda(R N G(V)) \leq \phi(V)$. It was recently proved in [17] that for any constant $\varepsilon>0$, it is asymptotically almost sure (abbreviated by a.a.s.) that

$$
(1-\varepsilon) \sqrt{\frac{\ln n}{\sigma n}} \leq \phi\left(\mathcal{P}_{n}\right) \leq(1+\varepsilon) \sqrt{\frac{\ln n}{\sigma n}} .
$$

As the immediate consequence of Theorem 1.1, a tighter a.a.s. lower bound on $\phi\left(\mathcal{P}_{n}\right)$ can be obtained: For any sequence $\left(c_{n}\right)$ satisfying that $\lim _{n \rightarrow \infty} c_{n}=\infty$ and $\lim _{n \rightarrow \infty} c_{n} / \ln n=0$, it is a.s.s. that

$$
\phi\left(\mathcal{P}_{n}\right) \geq \sqrt{\frac{\ln n-c_{n}}{\sigma n}} .
$$

In what follows, $\mathbf{o}$ is the origin of the Euclidean plane $\mathbb{R}^{2}$, and $\mathbb{D}$ is the unit-area (closed) disk centered at o. We assume that $\mathcal{P}_{n}$ is the Poisson point process over $\mathbb{D}$ with density $n$. We denote by $\mathcal{X}_{n}=\left(X_{1}, \ldots, X_{n}\right)$ the uniform $n$-point process over $\mathbb{D}$. The symbols $O, o, \sim$ always refer to the limit $n \rightarrow \infty$. To avoid trivialities, we tacitly assume $n$ to be sufficiently large if necessary. For simplicity of notation, the dependence of sets and random variables on $n$ will be frequently suppressed. For any finite set $S, \operatorname{card}(S)$ denotes the cardinality of $S$. For any set $S$ and positive integer $k$, the $k$-fold Cartesian product of $S$ is denoted by $S^{k}$. The Euclidean norm of a point $x$ is denoted by $\|x\|$, and the Euclidean distance between two points $u$ and $v$ is denoted by $\|u v\|$. The Lebesgue measure (or area) of a measurable set $A \subset \mathbb{R}^{2}$ is denoted by $|A|$. The topological boundary of a set $A \subset \mathbb{R}^{2}$ is denoted by $\partial A$. The open (respectively, closed) disk of radius $r$ centered at $x$ is denoted by $D(x, r)$ (respectively, $\bar{D}(x, r))$.

The remaining of this paper is organized as follows. The proof for Theorem 1.1 is presented in Sec. 3. Some preliminary results to be used in the proof for Theorem 1.1 are established in Sec. 2. The proofs for two technical lemmas on the limits of several relevant integrals are postponed in Sec. 4.

\section{Preliminaries}

The lune of a line segment $u v$, denoted by $L(u v)$, is the intersection of the disks $D(u,\|u v\|)$ and $D(v,\|u v\|)$ (see Fig. 1); the line segment $u v$ is called the waist of $L(u v)$; the two intersection points of $\partial D(u,\|u v\|)$ and $\partial D(v,\|u v\|)$ are called the vertices of $L(u v)$. It is easy to verify that

$$
|L(u v)|=\sigma\|u v\|^{2} .
$$

Thus, $\sigma$ is the area of the lune whose waist has unit length. For a finite planar set $V, R N G(V)$ consists of all edges $u v$ satisfying that $L(u v) \cap V=\emptyset$. For a finite 


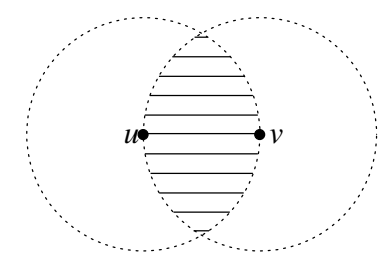

Fig. 1. The lune $L(u v)$ of a line segment $u v$.

planar set $V$ and any number $r>0$, the $r$-disk graph of $V$ consists of all edges $u v$ satisfying that $\|u v\| \leq r$.

For any line segment $e$, we define

$$
\nu(e)=|L(e) \cap \mathbb{D}| .
$$

A geometric graph is a graph on a finite planar set whose edges are line segments. Let $H$ be a geometric graph. We use $V(H)$ and $E(H)$ to denote the vertex set and edge set of $H$ respectively. Let $\chi(H)$ be the indicator for $H \subseteq R N G(V(H))$ and define

$$
\nu(H)=\left|\left(\cup_{e \in E(H)} L(e)\right) \cap \mathbb{D}\right| .
$$

For any positive number $n$, define

$$
\begin{aligned}
& f_{n}(H)=\chi(H) e^{-n \nu(H)}, \\
& g_{n}(H)=e^{-n \sum_{e \in E(H)} \nu(e)} .
\end{aligned}
$$

Let $\varepsilon=0.001$. Fix a sequence $\left(c_{n}\right)$ of real numbers satisfying that $c_{n}>c$, $c_{n}=o(\ln n)$ and $c_{n} \rightarrow \infty$. Let

$$
\begin{aligned}
& R_{n}=\sqrt{\frac{\ln n+c_{n}}{\sigma n}}, \\
& R_{n}^{\prime}=\left(1+\frac{\varepsilon}{2}\right) \sqrt{\frac{\ln n}{\sigma n}} .
\end{aligned}
$$

Then, for sufficiently large $n$, we have $r_{n}<R_{n}<R_{n}^{\prime}<(1+\varepsilon) r_{n}$. Define

$$
\begin{aligned}
\Omega & =\left\{\left(x_{1}, x_{2}\right) \in \mathbb{D}^{2}: r_{n}<\left\|x_{1} x_{2}\right\| \leq R_{n}\right\}, \\
\Omega^{\prime} & =\left\{\left(x_{1}, x_{2}\right) \in \mathbb{D}^{2}: R_{n}<\left\|x_{1} x_{2}\right\| \leq R_{n}^{\prime}\right\} .
\end{aligned}
$$

The following Lemma gives the limits of two fundamental integrals, whose proof is postponed to Sec. 4.

Lemma 2.1. The following are true:

$$
\begin{aligned}
& n^{2} \int_{\Omega} e^{-n v\left(x_{1} x_{2}\right)} d x_{1} d x_{2} \sim 2 \mu, \\
& n^{2} \int_{\Omega^{\prime}} e^{-n v\left(x_{1} x_{2}\right)} d x_{1} d x_{2}=o(1) .
\end{aligned}
$$


A topology with numbered vertices is specified by a collection of the pairs of the indices of the numbered vertices. For any integer $m \geq 2$, denote $\mathcal{T}_{m}$ set of topologies on $m$ numbered vertices without isolated vertex. For any $\tau \in \mathcal{T}_{m}$, and any sequence $U$ of $m$ planar points, $\tau(U)$ denotes the geometric graph on $U$ with topology $\tau$. For any $\tau \in \mathcal{T}_{m}$, we denote by $\Gamma(\tau)$ the set of $\mathbf{x} \in \mathbb{D}^{m}$ satisfying that all edges of $\tau(\mathbf{x})$ have length in $\left(r_{n}, R_{n}\right]$. Note that for each $\mathbf{x} \in \Gamma(\tau)$, the $\sqrt{3} R_{n}$-disk graph on the midpoints of the edges in any connected component of $\tau(\mathbf{x})$ is connected. Thus, the $\sqrt{3} R_{n}$-disk graph on the midpoints of the edges in $\tau(\mathbf{x})$ has no more connected components than $\tau(\mathbf{x})$ itself. For any positive integer $l$ no more than the number of connected components of $\tau$, we denote by $\Gamma_{l}(\tau)$ the set of $\mathbf{x} \in \Gamma(\tau)$ such that the $\sqrt{3} R_{n}$-disk graph on the midpoints of the edges in $\tau(\mathbf{x})$ has $l$ connected components.

Lemma 2.2. Suppose that $2<m \leq 2 k$ and $\tau$ is a forest in $\mathcal{T}_{m}$ with $k$ edges. Then, for any positive integer $l \leq \min \{m-k, k-1\}$,

$$
\begin{aligned}
& n^{m} \int_{\Gamma_{l}(\tau)} f_{n}(\tau(\mathbf{x})) d \mathbf{x}=o(1), \\
& n^{m} \int_{\Gamma_{l}(\tau)} g_{n}(\tau(\mathbf{x})) d \mathbf{x}=o(1) .
\end{aligned}
$$

The proof for Lemma 2.2 is also postponed to Sec. 4 . Lemma 2.2 implies the following two corollaries.

Corollary 2.3. Suppose that $\tau \in \mathcal{T}_{2 k}$ is a perfect matching for some $k \geq 2$. Then,

$$
n^{2 k} \int_{\Gamma_{k}(\tau)} g_{n}(\tau(\mathbf{x})) d \mathbf{x} \sim(2 \mu)^{k} .
$$

Proof. We denote by $C_{k}$ the perfect matching of $2 k$ numbered vertices $v_{1}, v_{2}, \ldots, v_{2 k}$ which consists of $k$ edges $v_{2 i-1} v_{2 i}$ for $1 \leq i \leq k$. By symmetry, we only have to prove the lemma holds for $\tau=C_{k}$. Note that

$$
\Gamma_{k}\left(C_{k}\right)=\Gamma\left(C_{k}\right) \backslash \cup_{l=1}^{k-1} \Gamma_{l}\left(C_{k}\right) .
$$

Since $\Gamma\left(C_{k}\right)=\Omega^{k}$, we have

$$
\begin{aligned}
n^{2 k} \int_{\Gamma\left(C_{k}\right)} g_{n}\left(C_{k}(\mathbf{x})\right) d \mathbf{x} & =\prod_{i=1}^{k}\left(n^{2} \int_{\Omega} e^{-n \nu\left(x_{2 i-1} x_{2 i}\right)} d x_{2 i-1} d x_{2 i}\right) \\
& \sim(2 \mu)^{k} .
\end{aligned}
$$

By Lemma 2.2 for each $1 \leq l \leq k-1$,

$$
n^{2 k} \int_{\Gamma_{l}\left(C_{k}\right)} g_{n}\left(C_{k}(\mathbf{x})\right) d \mathbf{x}=o(1) .
$$

Thus, the corollary holds. 
Corollary 2.4. Suppose that $\tau \in \mathcal{T}_{m}$ is not a perfect matching. Then,

$$
n^{m} \int_{\Gamma(\tau)} f_{n}(\tau(\mathbf{x})) d \mathbf{x}=o(1)
$$

Proof. Clearly, $m>2$. Let $\tau^{\prime}$ be a maximal spanning forest of $\tau$. Then, $\tau^{\prime} \in \mathcal{T}_{m}$ and $\tau^{\prime}$ is not a perfect matching. In addition, $\Gamma\left(\tau^{\prime}\right) \supseteq \Gamma(\tau)$, and for any $\mathbf{x} \in \Gamma(\tau)$,

$$
f_{n}\left(\tau^{\prime}(\mathbf{x})\right) \geq f_{n}(\tau(\mathbf{x}))
$$

Hence,

$$
\int_{\Gamma(\tau)} f_{n}(\tau(\mathbf{x})) d \mathbf{x} \leq \int_{\Gamma(\tau)} f_{n}\left(\tau^{\prime}(\mathbf{x})\right) d \mathbf{x} \leq \int_{\Gamma\left(\tau^{\prime}\right)} f_{n}\left(\tau^{\prime}(\mathbf{x})\right) d \mathbf{x} .
$$

Thus, it is sufficient to show that

$$
n^{m} \int_{\Gamma\left(\tau^{\prime}\right)} f_{n}\left(\tau^{\prime}(\mathbf{x})\right) d \mathbf{x}=o(1)
$$

Let $k$ be the number of edges in $\tau^{\prime}$. Then, $\tau$ has $m-k$ tree components and hence

$$
\Gamma\left(\tau^{\prime}\right)=\cup_{l=1}^{m-k} \Gamma_{l}\left(\tau^{\prime}\right)
$$

Since $\tau^{\prime}$ is not a perfect matching, we have $m<2 k$, which implies $m-k \leq k-1$. By Lemma 2.2, for any $1 \leq l \leq m-k$,

$$
n^{m} \int_{\Gamma_{l}\left(\tau^{\prime}\right)} f_{n}\left(\tau^{\prime}(\mathbf{x})\right) d \mathbf{x}=o(1) .
$$

Therefore,

$$
n^{m} \int_{\Gamma\left(\tau^{\prime}\right)} f_{n}\left(\tau^{\prime}(\mathbf{x})\right) d \mathbf{x}=\sum_{l=1}^{m-k} n^{m} \int_{\Gamma_{l}\left(\tau^{\prime}\right)} f_{n}\left(\tau^{\prime}(\mathbf{x})\right) d \mathbf{x}=o(1) .
$$

For any geometric graph $H$, let $B_{n}(H)$ be the indicator for $H \subseteq R N G(V(H) \cup$ $\left.\mathcal{P}_{n}\right)$ and all edges of $H$ have length in $\left(r_{n}, R_{n}\right]$.

Lemma 2.5. For any $\tau \in \mathcal{T}_{m}$, if $\tau$ is a perfect matching then

$$
n^{m} \mathbf{E}\left[B_{n}\left(\tau\left(\mathcal{X}_{m}\right)\right)\right] \sim(2 \mu)^{m / 2}
$$

otherwise,

$$
n^{m} \mathbf{E}\left[B_{n}\left(\tau\left(\mathcal{X}_{m}\right)\right)\right]=o(1)
$$

Proof. For any $\tau \in \mathcal{T}_{m}$,

$$
\mathbf{E}\left[B_{n}\left(\tau\left(\mathcal{X}_{m}\right)\right)\right]=\int_{\Gamma(\tau)} \operatorname{Pr}\left[B_{n}(\tau(\mathbf{x}))=1\right] d \mathbf{x} .
$$

For any $\mathbf{x} \in \Gamma(\tau)$,

$$
\operatorname{Pr}\left[B_{n}(\tau(\mathbf{x}))=1\right] \leq f_{n}(\tau(\mathbf{x}))
$$


In addition, if $\tau$ is a perfect matching then for any $\mathbf{x} \in \Gamma_{m / 2}(\tau)$,

$$
\operatorname{Pr}\left[B_{n}(\tau(\mathbf{x}))=1\right]=g_{n}(\tau(\mathbf{x}))
$$

If $\tau$ is not a perfect matching, then by Corollary 2.4, we have

$$
n^{m} \mathbf{E}\left[B_{n}\left(\tau\left(\mathcal{X}_{m}\right)\right)\right] \leq n^{m} \int_{\Gamma(\tau)} f_{n}(\tau(\mathbf{x})) d \mathbf{x}=o(1)
$$

In the next, we assume that $\tau$ is a perfect matching. Let $m=2 k$. For $k=1$, $\Gamma(\tau)=\Omega$ and hence by Lemma 2.1 we have

$$
\begin{aligned}
n^{2} \mathbf{E}\left[B_{n}\left(\tau\left(\mathcal{X}_{2}\right)\right)\right] & =n^{2} \int_{\Omega} \operatorname{Pr}\left[B_{n}(\tau(\mathbf{x}))=1\right] d \mathbf{x} \\
& =n^{2} \int_{\Omega} e^{-n \nu\left(x_{1} x_{2}\right)} d x_{1} d x_{2} \\
& \sim 2 \mu .
\end{aligned}
$$

So, the Lemma holds for $k=1$. So, we further assume that $k \geq 2$. Note that

$$
\begin{aligned}
n^{2 k} \mathbf{E}\left[B_{n}\left(\tau\left(\mathcal{X}_{2 k}\right)\right)\right] & =n^{2 k} \int_{\Gamma(\tau)} \operatorname{Pr}\left[B_{n}(\tau(\mathbf{x}))=1\right] d \mathbf{x} \\
& =\sum_{l=1}^{k} n^{2 k} \int_{\Gamma_{l}(\tau)} \operatorname{Pr}\left[B_{n}(\tau(\mathbf{x}))=1\right] d \mathbf{x} .
\end{aligned}
$$

By Corollary 2.3 we have

$$
n^{2 k} \int_{\Gamma_{k}(\tau)} \operatorname{Pr}\left[B_{n}(\tau(\mathbf{x}))=1\right] d \mathbf{x}=n^{2 k} \int_{\Gamma_{k}(\tau)} g_{n}(\tau(\mathbf{x})) d \mathbf{x} \sim(2 \mu)^{k},
$$

and for any $1 \leq l<k$,

$$
n^{2 k} \int_{\Gamma_{l}(\tau)} \operatorname{Pr}\left[B_{n}(\tau(\mathbf{x}))=1\right] d \mathbf{x} \leq n^{2 k} \int_{\Gamma_{l}(\tau)} f_{n}(\tau(\mathbf{x})) d \mathbf{x}=o(1) .
$$

Thus,

$$
n^{2 k} \mathbf{E}\left[B_{n}\left(\tau\left(\mathcal{X}_{2 k}\right)\right)\right] \sim(2 \mu)^{k}
$$

So, the Lemma holds in this case.

For any $\mathbf{x}=\left(x_{1}, x_{2}\right) \in \mathbb{D}^{2}$, let $B_{n}^{\prime}(\mathbf{x})$ be the indicator for $x_{1} x_{2} \in R N G\left(\left\{x_{1}, x_{2}\right\} \cup\right.$ $\left.\mathcal{P}_{n}\right)$ and $R_{n}<\left\|x_{1} x_{2}\right\| \leq R_{n}^{\prime}$.

Lemma 2.6. $n^{2} \mathbf{E}\left[B_{n}^{\prime}\left(\mathcal{X}_{2}\right)\right]=o(1)$ 
Proof. For any $\mathbf{x}=\left(x_{1}, x_{2}\right) \in \Omega^{\prime}$,

$$
\operatorname{Pr}\left[B_{n}^{\prime}(\mathbf{x})=1\right]=e^{-n v\left(x_{1} x_{2}\right)} .
$$

By Lemma 2.1,

$$
\begin{aligned}
n^{2} \mathbf{E}\left[B_{n}^{\prime}\left(\mathcal{X}_{2}\right)\right] & =n^{2} \int_{\Omega^{\prime}} \operatorname{Pr}\left[B_{n}^{\prime}(\mathbf{x})=1\right] d \mathbf{x} \\
& =\frac{n^{2}}{2} \int_{\Omega^{\prime}} e^{-n v\left(x_{1} x_{2}\right)} d x_{1} d x_{2} \\
& =o(1) .
\end{aligned}
$$

\section{Proof for Theorem 1.1}

We first give a brief overview on our approach to prove Theorem 1.1. Let $M_{n}$ (respectively, $M_{n}^{\prime}$ and $M_{n}^{\prime \prime}$ ) denote the number of edges in $R N G\left(\mathcal{P}_{n}\right)$ with length in $\left(r_{n}, R_{n}\right]$ (respectively, $\left(R_{n}, R_{n}^{\prime}\right]$ and $\left.\left(R_{n}^{\prime},+\infty\right)\right)$. Then, $\lambda\left(R N G\left(\mathcal{P}_{n}\right)\right) \leq r_{n}$ if and only if $M_{n}+M_{n}^{\prime}+M_{n}^{\prime \prime}=0$. In Lemma 3.1, we will show that $M_{n}^{\prime \prime}=0$ is a.a.s. In Lemma 3.4, we will prove that $E\left[M_{n}^{\prime}\right]=o(1)$, which implies that $M_{n}^{\prime}=0$ is a.a.s. by Markov's inequality. In Lemma 3.6, we will prove that $M_{n}$ is asymptotically Poisson with mean $\mu$. Consequently,

$$
\begin{aligned}
\lim _{n \rightarrow \infty} \operatorname{Pr}\left[\lambda\left(R N G\left(\mathcal{P}_{n}\right)\right) \leq r_{n}\right] & =\lim _{n \rightarrow \infty} \operatorname{Pr}\left[M_{n}+M_{n}^{\prime}+M_{n}^{\prime \prime}=0\right] \\
& =\lim _{n \rightarrow \infty} \operatorname{Pr}\left[M_{n}=0\right] \\
& =e^{-\mu} .
\end{aligned}
$$

We first utilize the tool of minimal scan statistics developed in [17] to prove that $M_{n}^{\prime \prime}=0$ is a.a.s.

Lemma 3.1. $\operatorname{Pr}\left[M_{n}^{\prime \prime}>0\right]=o(1)$.

Proof. For any finite point set $V \subset \mathbb{D}$ and any $r>0$, define

$$
\mathcal{S}(V, r)=\min _{\{u, v\} \subset \mathbb{D}, \mid u v \|=r}|V \cap L(u v)| .
$$

For any pair of $\{u, v\} \subset \mathbb{D}$ with $\|u v\|=R_{n}^{\prime}$,

$$
n|L(u v)|=n \cdot \sigma\left(R_{n}^{\prime}\right)^{2}=\left(1+\frac{\varepsilon}{2}\right)^{2} \ln n>(1+\varepsilon) \ln n .
$$

Using this fact and following the same argument in the proof of Lemma 9 in [17], we can prove that $\mathcal{S}\left(\mathcal{P}_{n}, R_{n}^{\prime}\right)>\Theta(\ln n)$ is a.a.s. In particular, $\mathcal{S}\left(\mathcal{P}_{n}, R_{n}^{\prime}\right)>0$ a.a.s. Next, we claim that the event $M_{n}^{\prime \prime}>0$ implies the event $\mathcal{S}\left(\mathcal{P}_{n}, R_{n}^{\prime}\right)=0$. Suppose that $M_{n}^{\prime \prime}>0$. Then there are a pair of nodes $\{X, Y\} \subset \mathcal{P}_{n}$ such that $\|X Y\|>R_{n}^{\prime}$ and $L(X Y) \cap \mathcal{P}_{n}$ is empty. Let $Y^{\prime}$ be the point on the line segment $X Y$ satisfying that $\left\|X Y^{\prime}\right\|=R_{n}^{\prime}$. Thus $L\left(X Y^{\prime}\right) \subset L(X Y)$. So $L\left(X Y^{\prime}\right) \cap \mathcal{P}_{n}$ is empty. This implies 
that $\mathcal{S}\left(\mathcal{P}_{n}, r_{n}\right)=0$. Therefore, our claim holds. Consequently,

$$
\operatorname{Pr}\left[M_{n}^{\prime \prime}>0\right] \leq \operatorname{Pr}\left[\mathcal{S}\left(\mathcal{P}_{n}, R_{n}^{\prime}\right)=0\right]=o(1) .
$$

Two key techniques used in our remaining proof are the Palm theory for Poisson processes (see, e.g. Theorem 1.6 in [12]) and the Brun's sieve (see, e.g. Theorem 10 in $[16])$, which are stated below.

Theorem 3.2. Suppose that $N$ is a non-negative integer random variable, and $B_{1}, \ldots, B_{N}$ are $N$ Bernoulli random variables. If there is a constant $\mu$ such that for every fixed positive integer $k$,

$$
\mathbf{E}\left[\sum_{I \subseteq\{1, \ldots, N\},|I|=k} \prod_{i \in I} B_{i}\right] \sim \frac{\mu^{k}}{k !}
$$

then $\sum_{i=1}^{N} B_{i}$ is asymptotically Poisson with mean $\mu$.

Theorem 3.3. Suppose that $h(U, V)$ is a bounded measurable function defined on all pairs of the form $(U, V)$ with $V$ being a finite planar set and $U$ being a subset of $V$. Then for any positive integer $k$,

$$
\mathbf{E}\left[\sum_{U \subseteq \mathcal{P}_{n},|U|=k} h\left(U, \mathcal{P}_{n}\right)\right]=\frac{n^{k}}{k !} \mathbf{E}\left[h\left(\mathcal{X}_{k}, \mathcal{X}_{k} \cup \mathcal{P}_{n}\right)\right] .
$$

Now, we apply Palm theory to show that $E\left[M_{n}^{\prime}\right]$ is vanishing.

Lemma 3.4. $E\left[M_{n}^{\prime}\right]=o(1)$.

Proof. For any pair $(U, V)$ with $V$ being a finite planar set and $U$ being a subset of $V$, define $h^{\prime}(U, V)$ to be the number of edges in the subgraph of $R N G(V)$ induced by $U$ with length in $\left(R_{n}, R_{n}^{\prime}\right]$. By applying the Palm theory, we have

$$
\begin{aligned}
\mathbf{E}\left[M_{n}^{\prime}\right] & =\mathbf{E}\left[\sum_{U \subset \mathcal{P}_{n},|U|=2} h^{\prime}\left(U, \mathcal{P}_{n}\right)\right] \\
& =\frac{n^{2}}{2} \mathbf{E}\left[h^{\prime}\left(\mathcal{X}_{2}, \mathcal{X}_{2} \cup \mathcal{P}_{n}\right)\right] \\
& =\frac{n^{2}}{2} \mathbf{E}\left[B_{n}^{\prime}\left(\mathcal{X}_{2}\right)\right] .
\end{aligned}
$$

By Lemma 2.6, the lemma follows.

For any positive integer $k$, denote by $\mathcal{H}_{n, k}$ the collection of $k$-edge subgraphs of $R N G\left(\mathcal{P}_{n}\right)$ in which all edges have length in $\left(r_{n}, R_{n}\right]$ and no vertex is isolated. Next, we apply Palm theory to compute the asymptotic average of $\operatorname{card}\left(\mathcal{H}_{n, k}\right)$. 
Lemma 3.5. For any fixed positive integer $k$,

$$
\mathbf{E}\left[\operatorname{card}\left(\mathcal{H}_{n, k}\right)\right] \sim \frac{\mu^{k}}{k !}
$$

Proof. For any pair $(U, V)$ with $V$ being a finite planar set and $U$ being a subset of $V$, define $h(U, V)$ to be the number of $k$-edge subgraphs of $R N G(V)$ on $U$ in which all edges have length in $\left(r_{n}, R_{n}\right]$ and no vertex is isolated. By applying the Palm theory, we have

$$
\begin{aligned}
\mathbf{E}\left[\operatorname{card}\left(\mathcal{H}_{n}\right)\right] & =\mathbf{E}\left[\sum_{m=2}^{2 k} \sum_{U \subset \mathcal{P}_{n},|U|=m} h\left(U, \mathcal{P}_{n}\right)\right] \\
& =\sum_{m=2}^{2 k} \mathbf{E}\left[\sum_{U \subset \mathcal{P}_{n},|U|=m} h\left(U, \mathcal{P}_{n}\right)\right] \\
& =\sum_{m=2}^{2 k} \frac{n^{m}}{m !} \mathbf{E}\left[h\left(\mathcal{X}_{m}, \mathcal{X}_{m} \cup \mathcal{P}_{n}\right)\right]
\end{aligned}
$$

Thus, it is sufficient to show that

$$
\frac{n^{m}}{m !} \mathbf{E}\left[h\left(\mathcal{X}_{m}, \mathcal{X}_{m} \cup \mathcal{P}_{n}\right)\right]= \begin{cases}0, & \text { if } 2 \leq m<2 k \\ \frac{\mu^{k}}{k !}, & \text { if } m=2 k .\end{cases}
$$

For any positive integer $k$ and any $2 \leq m \leq 2 k$, denote $\mathcal{T}_{m, k}$ the set of topologies in $\mathcal{T}_{m}$ with exactly $k$ edges. Note that any topology in $\mathcal{T}_{2 k, k}$ is a perfect matching, and

$$
\operatorname{card}\left(\mathcal{T}_{2 k, k}\right)=\frac{1}{k !}\left(\begin{array}{c}
2 k \\
2,2, \ldots, 2
\end{array}\right)=\frac{(2 k) !}{k ! 2^{k}} .
$$

By Lemma 2.5, we have

$$
\begin{aligned}
\frac{n^{2 k}}{(2 k) !} \mathbf{E}\left[h\left(\mathcal{X}_{2 k}, \mathcal{X}_{2 k} \cup \mathcal{P}_{n}\right)\right] & =\frac{1}{(2 k) !} \sum_{\tau \in \mathcal{T}_{2 k, k}} n^{2 k} \mathbf{E}\left[B\left(\tau\left(\mathcal{X}_{2 k}\right)\right)\right] \\
& \sim \frac{1}{(2 k) !} \cdot \frac{(2 k) !}{k ! 2^{k}} \cdot(2 \mu)^{k} \\
& =\frac{\mu^{k}}{k !} .
\end{aligned}
$$

Now suppose that $2 \leq m<2 k$. Then any topology in $\mathcal{T}_{m, k}$ is not a perfect matching. By Lemma 2.5,

$$
n^{m} \mathbf{E}\left[h\left(\mathcal{X}_{m}, \mathcal{X}_{m} \cup \mathcal{P}_{n}\right)\right]=\sum_{\tau \in \mathcal{T}_{m, k}} n^{m} \mathbf{E}\left[B_{n}\left(\tau\left(\mathcal{X}_{m}\right)\right)\right]=o(1) .
$$

Finally, we apply the Brun's sieve together with Lemma 3.5 to prove $M_{n}$ is asymptotically Poisson. 
Lemma 3.6. $M_{n}$ is asymptotically Poisson with mean $\mu$.

Proof. Let $\mathcal{E}_{n}$ be the set of edges of $R N G\left(\mathcal{P}_{n}\right)$. For any edge $e \in \mathcal{E}_{n}$, define $\bar{B}(e)$ to be the Bernoulli random variable which equals to one if and only if $r_{n}<\|e\| \leq$ $R_{n}$. Then

$$
M_{n}=\sum_{e \in \mathcal{E}_{n}} \bar{B}(e) .
$$

For subset $F$ of $\mathcal{E}_{n}, \prod_{e \in F} \bar{B}(e)=1$ if and only if $F$ is the edge set of a subgraph of $R N G\left(\mathcal{P}_{n}\right)$ in which all edges have length in $\left(r_{n}, R_{n}\right]$ and no vertex is isolated. Fix a positive integer $k$. By treating each $k$-subset $F$ of $\mathcal{E}_{n}$ as an $k$-edge subgraphs of $R N G\left(\mathcal{P}_{n}\right)$, we have that

$$
\sum_{F \subseteq \mathcal{E}_{n},|F|=k} \prod_{e \in F} \bar{B}(e)=\operatorname{card}\left(\mathcal{H}_{n, k}\right) .
$$

Hence, by Lemma 3.5,

$$
\mathbf{E}\left[\sum_{F \subseteq \mathcal{E}_{n},|F|=k} \prod_{e \in F} \bar{B}(e)\right] \sim \frac{\mu^{k}}{k !} .
$$

By Theorem 3.2, $M_{n}$ is asymptotically Poisson with mean $\mu$.

\section{Proofs for Lemma 2.1 and Lemma 2.2}

In this section, we prove Lemma 2.1 and Lemma 2.2. We first present some useful geometric results. For $x \in \mathbb{D}$, let $t(x)$ denote the distance between $x$ and $\partial \mathbb{D}$, which is equal to $\frac{1}{\sqrt{\pi}}-\|x\|$. For any $0<\rho<\frac{1}{\sqrt{\pi}}$, define

$$
\begin{aligned}
& \mathbb{D}_{\rho}(0)=\{x \in \mathbb{D}: t(x) \geq \rho\}, \\
& \mathbb{D}_{\rho}(1)=\left\{x \in \mathbb{D}: \sqrt{\frac{1}{\pi}-\rho^{2}} \leq t(x)<\rho\right\}, \\
& \mathbb{D}_{\rho}(2)=\left\{x \in \mathbb{D}: t(x)<\sqrt{\frac{1}{\pi}-\rho^{2}}\right\} .
\end{aligned}
$$

With this notation, the midpoint of any line segment $x y \subset \mathbb{D}$ is not in $\mathbb{D}_{\|x y\| / 2}(2)$. For $x \in \mathbb{D}$ and $0<\rho<\frac{1}{\sqrt{\pi}}$, define $\theta(x, \rho)$ as follows. If $x \in \mathbb{D}_{\rho}(0)$, then $\theta(x, \rho)=2 \pi$. If $x \in \mathbb{D}_{\rho}(2)$, then $\theta(x, \rho)=0$. If $x \in \mathbb{D}_{\rho}(1)$, let $u$ and $v$ be the two intersection points of $\partial B(x, \rho)$ and $\partial \mathbb{D}$, and define $\theta(x, \rho)=2 \pi-\angle u x v$ (see Fig. 2).

Lemma 4.1. For $x \in \mathbb{D}$ and $0<\rho<\frac{1}{\sqrt{\pi}}$,

$$
\rho \theta(x, \rho) \leq 2 \pi t(x) .
$$




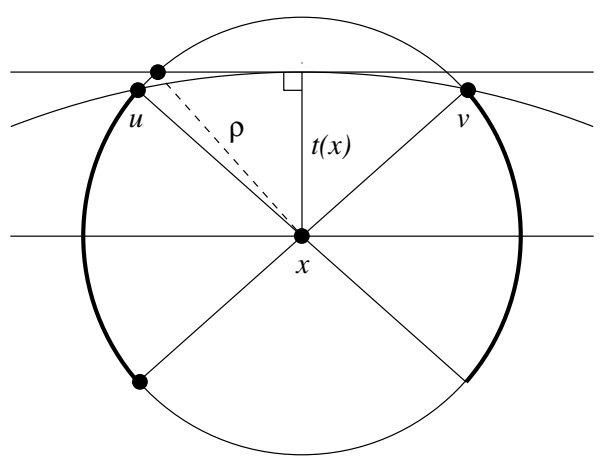

Fig. 2. If $x \in \mathbb{D}_{\rho}(1)$, then $\theta(x, \rho)=2 \pi-2 \angle u x v$.

Proof. The lemma holds trivially if $x \in \mathbb{D}_{\rho}(0)$ or $x \in \mathbb{D}_{\rho}(2)$. So, we consider the case that $x \in \mathbb{D}_{\rho}(1)$. It is easy to see that

$$
\theta(x, \rho) \leq 4 \arcsin \frac{t(x)}{\rho} .
$$

Using the equality $\sin \alpha \geq \frac{2}{\pi} \alpha$ for any $\alpha \in[0, \pi / 2]$, we obtain

$$
\theta(x, \rho) \leq 4 \cdot \frac{\pi}{2} \cdot \frac{t(x)}{\rho}=\frac{2 \pi t(x)}{\rho} .
$$

Thus, $\rho \theta(x, \rho) \leq 2 \pi t(x)$.

If $e \subset \mathbb{D}$ and the midpoint of $e$ is apart from $\partial \mathbb{D}$ by at least $\frac{\sqrt{3}}{2}\|e\|$, then $L(e) \subset \mathbb{D}$. The next lemma gives a lower bound on $|L(e) \cap \mathbb{D}|$ if otherwise.

Lemma 4.2. Consider a line segment $e \subset \mathbb{D}$ with midpoint $z$. If $t(z) \leq \frac{\sqrt{3}}{2}\|e\|$, then

$$
|L(e) \cap \mathbb{D}| \geq \frac{1}{2}|L(e)|+\frac{\|e\|}{2} t(z) .
$$

Proof. Let $a$ and $b$ be the two endpoints of $e$, and $c_{1}$ and $c_{2}$ be the two vertices of $L(e)$ with $c_{1}$ being farther away from the center of $\mathbb{D}$ (see Fig. 3). Then, the half lune $a b c_{2}$ is fully contained in $\mathbb{D}$. If $c_{1} \in \partial \mathbb{D}$, then the triangle $a b c_{1}$ is contained in $\mathbb{D}$ and its area is $\frac{\|e\|}{2}\|a z\| \geq \frac{\|e\|}{2} t(z)$. So, the lemma holds if $c_{1} \in \partial \mathbb{D}$. Now assume that $c_{1} \notin \partial \mathbb{D}$. Let $u$ be the intersection point of $c_{1} z$ and $\partial \mathbb{D}$. Then, the triangle $a b u$ is contained in $\mathbb{D}$ and its area is $\frac{\|e\|}{2}\|u z\| \geq \frac{\|e\|}{2} t(z)$. So, the lemma also holds if $c_{1} \notin \partial \mathbb{D}$.

For any geometric graph $H$ on a finite subset of $\mathbb{D}$, an edge $e$ of $H$ is called an outermost edge of $H$ if its midpoint is the nearest to $\partial \mathbb{D}$.

Lemma 4.3. Suppose that $H$ is a geometric graph over a finite subset of $\mathbb{D}$ with at least two edges satisfying that all the edges have length between $R /(1+\varepsilon)$ and $R$ for 


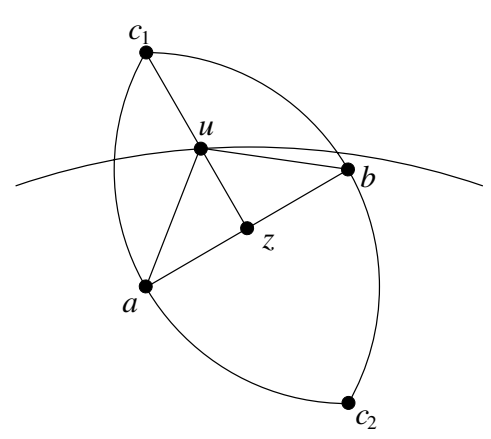

Fig. 3. $L(e) \cap \mathbb{D}$ contains the triangle $a b c_{2}$ and the half lune $a b u$.

$R \leq \frac{1}{200 \sqrt{\pi}}$ and the midpoints of its edges induce a connected $\sqrt{3} R$-disk graph. Let $e^{\prime}$ be an outermost edge of $H$, and $\ell$ be the largest distance between the midpoint of $e^{\prime}$ and the midpoints of other edges of $H$. Then,

$$
\sum_{e \in E(H)} \nu(e) \geq \nu\left(e^{\prime}\right)+0.0026 R \ell .
$$

In addition, if $\chi(H)=1$ then

$$
\nu(H) \geq \nu\left(e^{\prime}\right)+0.0026 R \ell .
$$

The proof of this lemma is very lengthy and complicated. We omit the proof due to the limitation on the space.

Now it is ready to give the proofs for Lemma 2.1 and Lemma 2.2. We will frequently change the integral variables using a technique introduced in [16]. Consider a tree topology on $k$ planar points $x_{1}, x_{2}, \ldots, x_{k}$, and assume without loss of generality that $x_{k-1} x_{k}$ is an edge in this tree. Let $z_{k-1}, \rho$, and $\omega$ be the midpoint, half-length and the slope of $x_{k-1} x_{k}$ respectively. We root the tree at $x_{k}$. For $1 \leq i \leq k-2$, let $z_{i}$ be the midpoint of the edge between $x_{i}$ and its parent in such rooted tree. Then, we replace $x_{1}, x_{2}, \ldots, x_{k}$ by $z_{1}, \ldots, z_{k-1}, \rho, \omega$. The Jacobian determinant of this change is $4^{k-1} \rho$.

We begin with the proof for Lemma 2.1. Let $\rho=\rho\left(x_{1}, x_{2}\right)$ be the half-length of $x_{1} x_{2}$, and $z=z\left(x_{1}, x_{2}\right)$ be the midpoint of $x_{1} x_{2}$. Let $\Omega_{1}$ be the set of $\left(x_{1}, x_{2}\right) \in \Omega$ satisfying that $z \in \mathbb{D}_{\sqrt{3} \rho}(0)$, and let $\Omega_{2}=\Omega \backslash \Omega_{1}$. First, we calculate the integration over $\Omega_{1}$. If $\left(x_{1}, x_{2}\right) \in \Omega_{1}, L\left(x_{1} x_{2}\right)$ is fully contained in $\mathbb{D}$ and $v\left(x_{1} x_{2}\right)=4 \sigma \rho^{2}$. Changing the integration variable $x_{1}$ and $x_{2}$ by $z, \rho$, and the slope of $x_{1} x_{2}$ yields

$$
\begin{aligned}
n^{2} \int_{\Omega_{1}} e^{-n v\left(x_{1} x_{2}\right)} d x_{1} d x_{2} & =8 \pi n^{2} \int_{\frac{r_{n}}{2}}^{\frac{R_{n}}{2}} e^{-4 n \sigma \rho^{2}} \rho d \rho \int_{\mathbb{D}_{\sqrt{3} \rho}(0)} d z \\
& \sim 8 \pi n^{2} \int_{\frac{r_{n}}{2}}^{\frac{R_{n}}{2}} e^{-4 n \sigma \rho^{2}} \rho d \rho
\end{aligned}
$$


38 P.-J. Wan et al.

$$
\begin{aligned}
& =-\frac{\pi}{\sigma} n e^{-4 \sigma \rho^{2}} \mid \frac{\frac{R_{n}}{2}}{\frac{r_{n}}{2}} \\
& \sim \frac{\pi}{\sigma} e^{-c} \\
& =2 \mu .
\end{aligned}
$$

Next, we calculate the integration over $\Omega_{2}$. Let $t=t(z)$ be the distance between $z$ and $\partial \mathbb{D}$. By Lemmas 4.1 and 4.2 , we have

$$
\rho \theta(z, \rho) \leq 2 \pi t
$$

and

$$
v\left(x_{1} x_{2}\right) \geq 2 \sigma \rho^{2}+\rho t
$$

Changing the integration variable as above yields

$$
\begin{aligned}
n^{2} \int_{\Omega_{2}} e^{-n v\left(x_{1} x_{2}\right)} d x_{1} d x_{2} & \leq n^{2} \int_{\Omega_{2}} e^{-n\left(2 \sigma \rho^{2}+\rho t\right)} d x_{1} d x_{2} \\
& =4 n^{2} \int_{\frac{r_{n}}{2}}^{\frac{R_{n}}{2}} d \rho \int_{\mathbb{D}_{\sqrt{3} \rho}(1) \backslash \mathbb{D}_{\rho}(2)} e^{-n\left(2 \sigma \rho^{2}+\rho t\right)} \rho \theta(z, \rho) d z \\
& =4 n^{2} \int_{\frac{r_{n}}{2}}^{\frac{R_{n}}{2}} d \rho \int_{\mathbb{D}_{\sqrt{3} \rho}(1) \backslash \mathbb{D}_{\rho}(2)} e^{-n\left(2 \sigma \rho^{2}+\rho t\right)} \rho \theta(z, \rho) d z \\
& \leq 8 \pi n^{2} e^{-2 n \sigma \rho^{2}} \int_{\frac{r_{n}}{2}}^{\frac{R_{n}}{2}} d \rho \int_{\mathbb{D}} e^{-n \rho t} t d z \\
& =O(1) n^{1.5} \int_{\frac{r_{n}}{2}}^{\frac{R_{n}}{2}} d \rho \int_{0}^{1 / \sqrt{\pi}} e^{-n \rho t} t d t \\
& \leq O(1) n^{1.5} \int_{\frac{r_{n}}{2}}^{\frac{R_{n}}{2}} d \rho \int_{0}^{\infty} e^{-n \rho t} t d t \\
& =O(1) . \\
& =O(1) \frac{1}{\sqrt{n}} \int_{\frac{r_{n}}{2}}^{\frac{R_{n}}{2}} \rho^{-2} d \rho \\
& \leq O(1) \frac{1}{\sqrt{n}} r_{n}^{-2} R_{n} \\
& =O(1) \frac{1}{\sqrt{n} r_{n}} \\
& \\
& =O(1) \\
& \\
& \\
& \\
&
\end{aligned}
$$


Therefore,

$$
n^{2} \int_{\Omega} e^{-n v_{x_{1} x_{2}}} d x_{1} d x_{2} \sim 2 \mu .
$$

Note that $\Omega \cup \Omega^{\prime}$ consists of $\left(x_{1}, x_{2}\right) \in \mathbb{D}^{2}$ satisfying that $r_{n}<\left\|x_{1} x_{2}\right\| \leq R_{n}^{\prime}$. Using the same argument as above, we can show that

$$
n^{2} \int_{\Omega \cup \Omega^{\prime}} e^{-n v\left(x_{1} x_{2}\right)} d x_{1} d x_{2} \sim 2 \mu .
$$

Thus, the second asymptotic equality in Lemma 2.1 holds.

We move to prove Lemma 2.2 by establishing the following two lemmas.

Lemma 4.4. Suppose that $2<m \leq 2 k$ and $\tau$ is a forest in $\mathcal{T}_{m}$ with $k$ edges. Then,

$$
\begin{aligned}
& n^{m} \int_{\Gamma_{1}(\tau)} f_{n}(\tau(\mathbf{x})) d \mathbf{x}=o(1), \\
& n^{m} \int_{\Gamma_{1}(\tau)} g_{n}(\tau(\mathbf{x})) d \mathbf{x}=o(1) .
\end{aligned}
$$

Proof. Enumerate the edges of $\tau$ arbitrarily by $e_{1}, \ldots, e_{k}$. For any $\mathbf{x} \in \Gamma_{1}(\tau)$, let $z_{i}$ denote the middle point of $e_{i}$ in $F(\mathbf{x})$ for each $1 \leq i \leq k$. For any pair of distinct integers $p$ and $q$ between 1 and $k$, let $S_{p q}$ denote the set of $\mathbf{x} \in \Gamma_{1}(\tau)$ satisfying that $e_{p}$ is an outermost edge in $\tau(\mathbf{x})$ and $z_{q}$ is the farthest from $z_{p}$ among all $z_{1}, \ldots, z_{k}$. Then, it suffices to prove for any such $p$ and $q$,

$$
\begin{aligned}
& n^{m} \int_{S_{p q}} f_{n}(\tau(\mathbf{x})) d \mathbf{x}=o(1), \\
& n^{m} \int_{S_{p q}} g_{n}(\tau(\mathbf{x})) d \mathbf{x}=o(1) .
\end{aligned}
$$

Fix a pair of distinct integers $p$ and $q$ between 1 and $k$. Let $p^{\prime}$ and $p^{\prime \prime}$ be the indices of the two endpoints of the edges $e_{p}$. By Lemma 4.3 for any $\mathbf{x} \in S_{p q}$,

$$
\begin{aligned}
& f_{n}(\tau(\mathbf{x})) \leq e^{-n\left(v\left(x_{p^{\prime}} x_{p^{\prime \prime}}\right)+\eta R_{n}\left\|z_{p} z_{q}\right\|\right)}, \\
& g_{n}(\tau(\mathbf{x})) \leq e^{-n\left(v\left(x_{p^{\prime}} x_{p^{\prime \prime}}\right)+\eta R_{n}\left\|z_{p} z_{q}\right\|\right)},
\end{aligned}
$$

in which $\eta$ is a positive constant. Thus, we only need to show that

$$
n^{m} \int_{S_{p q}} e^{-n\left(v\left(x_{p^{\prime}} x_{p^{\prime \prime}}\right)+\eta R_{n}\left\|z_{p} z_{q}\right\|\right)} d \mathbf{x}=o(1) .
$$

We change the integral variables $x_{1}, \ldots, x_{m}$ as follows. For the tree component containing $e_{p}$, we replace the $x_{i}$ 's in this tree by the midpoints of the edges in this tree except $z_{p}$ and $x_{p^{\prime}}, x_{p^{\prime \prime}}$ (both of which are kept). For any other tree component, we use the method introduced at the beginning of this section: pick an arbitrary edge as the rooted edge. We replace $x_{i}$ 's in this tree by the midpoints of all the 
edges in this tree together with the half-length and slope of the root edge. Such change of integration variables yields

$$
\begin{aligned}
& n^{m} \int_{S_{p q}} e^{-n\left(v\left(x_{p^{\prime}} x_{p^{\prime \prime}}\right)+\eta R_{n}\left\|z_{p} z_{q}\right\|\right)} d \mathbf{x} \\
& \leq O(1) n^{m}\left(\int_{\Omega} e^{-n v v\left(x_{p^{\prime}} x_{p^{\prime \prime}}\right)} d x_{p^{\prime}} d x_{p^{\prime \prime}}\right)\left(\int_{\frac{r_{n}}{2}}^{\frac{R_{n}}{2}} \rho d \rho\right)^{m-k-1} \\
& \cdot\left(\int_{\mathbb{R}^{2}} e^{-\eta n R_{n}\left\|z_{p} z_{q}\right\|} d z_{q}\right)\left(\int_{\bar{D}\left(z_{p},\left\|z_{p} z_{q}\right\|\right)} d z\right)^{k-2} \\
& \sim O(1) n^{m-2}\left(R_{n}^{2}-r_{n}^{2}\right)^{m-k-1} \int_{\mathbb{R}^{2}} e^{-\eta n R_{n}\left\|z_{p} z_{q}\right\|}\left\|z_{p} z_{q}\right\|^{2(k-2)} d z_{q} \\
& \leq O(1) n^{m-2}\left(R_{n}^{2}-r_{n}^{2}\right)^{m-k-1} \int_{0}^{\infty} e^{-\eta n R_{n} \mu} \mu^{2 k-3} d \mu \\
&= O(1) \frac{n^{m-2}\left(R_{n}^{2}-r_{n}^{2}\right)^{m-k-1}}{\left(n R_{n}\right)^{2(k-1)}} \\
&= O(1) \frac{\left(n R_{n}^{2}-n r_{n}^{2}\right)^{m-k-1}}{\left(n R_{n}^{2}\right)^{k-1}} \\
&= O(1) \frac{\left(c_{n}-c\right)^{m-k-1}}{(\ln n)^{k-1}} \\
&= O(1), \quad
\end{aligned}
$$

where the asymptotic equality follows from Lemma 2.1, and the last equality follows from $c_{n}=o(\ln n)$ and $k \geq 2$.

Lemma 4.5. Suppose that $2<m \leq 2 k$ and $\tau$ is a forest in $\mathcal{T}_{m}$ with $k$ edges. For any integer $2 \leq l \leq \min \{m-k, k-1\}$,

$$
\begin{aligned}
& n^{m} \int_{\Gamma_{l}(\tau)} f_{n}(\tau(\mathbf{x})) d \mathbf{x}=o(1), \\
& n^{m} \int_{\Gamma_{l}(\tau)} g_{n}(\tau(\mathbf{x})) d \mathbf{x}=o(1) .
\end{aligned}
$$

Proof. $\tau$ has $m-k$ tree components on $m$ numbered vertices $v_{1}, v_{2}, \ldots, v_{m}$. Enumerate them arbitrarily by $T_{1}, \ldots, T_{m-k}$. For each $1 \leq q \leq m-k$, let

$$
I_{q}=\left\{1 \leq i \leq m: v_{i} \text { is a vertex of } T_{q}\right\} .
$$

Fix an integer $2 \leq l \leq \min \{m-k, k-1\}$. Consider any nontrivial $l$-partition $\Pi=$ $\left\{Q_{1}, Q_{2}, \ldots, Q_{l}\right\}$ of $\{1,2, \ldots, m-k\}$. It induces a partition $\Pi^{\prime}=\left\{P_{1}, P_{2}, \ldots, P_{l}\right\}$ of 
$\{1,2, \ldots, m\}$ in which $P_{j}=\cup_{q \in Q_{j}} I_{q}$ for each $1 \leq j \leq l$. Let $S(\Pi)$ denote the set of $\mathbf{x} \in \Gamma_{l}(\tau)$ such that for each $1 \leq j \leq l$, the set of midpoints of the subgraph of $\tau(\mathbf{x})$ induced by $\left\{x_{i}: i \in P_{j}\right\}$ is a connected component of the $\sqrt{3} R_{n}$-disk graph on the midpoints of the edges in $\tau(\mathbf{x})$. Then $\Gamma_{l}(\tau)$ is the union of $S(\Pi)$ over all nontrivial $l$-partitions $\Pi$ of $\{1,2, \ldots, m-k\}$. So, it is sufficient to show that for any $l$-partition $\Pi$ of $\{1,2, \ldots, k\}$,

$$
\begin{aligned}
& n^{m} \int_{S(\Pi)} f_{n}(\tau(\mathbf{x})) d \mathbf{x}=o(1), \\
& n^{m} \int_{S(\Pi)} g_{n}(\tau(\mathbf{x})) d \mathbf{x}=o(1) .
\end{aligned}
$$

Now, fix an $l$-partition $\Pi=\left\{Q_{1}, Q_{2}, \ldots, Q_{l}\right\}$ of $\{1,2, \ldots, m-k\}$. Let $\Pi^{\prime}=$ $\left\{P_{1}, P_{2}, \ldots, P_{l}\right\}$ be the partition of $\{1,2, \ldots, m\}$ induced by $\Pi$. For each $1 \leq j \leq l$, let $m_{j}=\operatorname{card}\left(P_{j}\right), \tau_{j}$ be the topology on $m_{j}$ numbered vertices which is a subgraph of $\tau$ induced by the subset of vertices $\left\{v_{i}: i \in P_{j}\right\}$. Then, at least one $m_{j} \geq 2$. For any $\mathbf{x}=\left(x_{1}, x_{2}, \ldots, x_{m}\right) \in \mathbb{D}^{m}$ and each $1 \leq j \leq l$, let

$$
\mathbf{x}^{(j)}=\left(x_{i_{1}}, x_{i_{2}}, \ldots, x_{i_{m_{j}}}\right)
$$

where $i_{1}, i_{2}, \ldots, i_{m_{j}}$ are the $m_{j}$ indices in $P_{j}$ in the increasing order. Clearly, for each $\mathbf{x} \in S(\Pi)$ and each $1 \leq j \leq l, \mathbf{x}^{(j)} \in \Gamma_{1}\left(\tau_{j}\right)$. Hence,

$$
S(\Pi) \subseteq\left\{\mathbf{x} \in \mathbb{D}^{m}: \mathbf{x}^{(j)} \in \Gamma_{1}\left(\tau_{j}\right), 1 \leq j \leq l\right\} .
$$

For any $\mathbf{x} \in S(\Pi)$,

$$
\begin{aligned}
\nu(\tau(\mathbf{x})) & =\sum_{j=1}^{l} \nu\left(\tau_{j}\left(\mathbf{x}^{(j)}\right)\right), \\
\chi(\tau(\mathbf{x})) & \leq \prod_{j=1}^{l} \chi\left(\tau_{j}\left(\mathbf{x}^{(j)}\right)\right),
\end{aligned}
$$

which imply

$$
f_{n}(\tau(\mathbf{x})) \leq \prod_{j=1}^{l} f_{n}\left(\tau_{j}\left(\mathbf{x}^{(j)}\right)\right) .
$$

It is obvious that for any $\mathbf{x} \in S(\Pi)$,

$$
g_{n}(\tau(\mathbf{x}))=\prod_{j=1}^{l} g_{n} t\left(\tau_{j}\left(\mathbf{x}^{(j)}\right)\right) .
$$


Thus,

$$
\begin{aligned}
n^{m} \int_{S(\Pi)} f_{n}(\tau(\mathbf{x})) d \mathbf{x} & \leq n^{m} \int_{S(\Pi)} \prod_{j=1}^{l} f_{n}\left(\tau_{j}\left(\mathbf{x}^{(j)}\right)\right) d \mathbf{x} \\
& \leq n^{m} \int_{\left\{\mathbf{x} \in \mathbb{D}^{m}: \mathbf{x}^{(j)} \in \Gamma_{1}\left(\tau_{j}\right), 1 \leq j \leq l\right\}} \prod_{j=1}^{l} f_{n}\left(\tau_{j}\left(\mathbf{x}^{(j)}\right)\right) d \mathbf{x} \\
& =\prod_{j=1}^{l}\left(n^{m_{j}} \int_{\Gamma_{1}\left(\tau_{j}\right)} f_{n}\left(\tau_{j}\left(\mathbf{x}^{(j)}\right)\right) d \mathbf{x}^{(j)}\right) \\
& =o(1)
\end{aligned}
$$

where the last equality follows from Lemma 2.1, Lemma 4.4 and the fact that at least one $m_{j} \geq 2$. Similarly, we can show that

$$
n^{m} \int_{S(\Pi)} g_{n}(\tau(\mathbf{x})) d \mathbf{x}=o(1) .
$$

So, the Lemma follows.

\section{Acknowledgment}

This work was supported in part by National Science Foundation of USA under grant CNS-0831831, Research Grants Council of Hong Kong SAR under Project No. CityU 122807 and CityU 114307, National Basic Research Program of China Grant 2007CB807900 and 2007CB807901, the NSC Grant No. NSC95-2221-E-009059-MY3 and NSC97-2221-E-009-052-MY3, ITRI Grant No. 83522N2000, and the MOE ATU Program.

\section{References}

[1] J. Cartigny, F. Ingelrest, D. Simplot-Ryl and I. Stojmenovic, Localized LMST and RNG based minimum energy broadcast protocols in ad hoc networks, IEEE INFOCOM (2003); also appeared in Ad Hoc Networks 3 (2005) 1-16.

[2] H. Dette and N. Henze, The limit distribution of the largest nearest-neighbour link in the unit $d$-cube, J. Appl. Probab. 26 (1989) 67-80.

[3] G. Finn, Routing and addressing problems in large metropolitan-scale internetworks, technical Report ISI Research Report ISU/RR-87-180, March 1987.

[4] K. Gabriel and R. Sokal, A new statistical approach to geographic variation analysis, Syst. Zool. 18 (1969) 259-278.

[5] P. Gupta and P. R. Kumar, Critical power for asymptotic connectivity in wireless networks, in Stochastic Analysis, Control, Optimization and Applications: A Volume in Honor of W. H. Fleming, eds. W. M. McEneaney, G. Yin and Q. Zhang (Birkhauser, March 1998), pp. 547-566.

[6] A. A.-K. Jeng and R.-H. Jan, The $r$-neighborhood graph: An adjustable structure for topology control in wireless ad hoc networks, IEEE Trans. Parall. Distri. Syst. 18 (2007) $536-549$. 
[7] B. Karp and H. T. Kung, GPSR: Greedy perimeter stateless routing for wireless networks, ACM MOBICOM, 2000.

[8] X.-Y. Li, P.-J. Wan and W. Yu, Power efficient and sparse spanner for wireless ad hoc networks, IEEE ICCCN, 2001.

[9] N. Li and J. C. Hou, Localized topology control algorithms for heterogeneous wireless networks, IEEE/ACM Trans. Netw. 13 (2005) 1313-1324.

[10] G. Kozma, Z. Lotker, M. Sharir and G. Stupp, Geometrically aware communication in random wireless networks, ACM PODC, 2004.

[11] M. D. Penrose, The longest edge of the random minimal spanning tree, Ann. Appl. Probab. 7 (1997) 340-361.

[12] M. Penrose, Random Geometric Graphs (Oxford University Press, 2003).

[13] M. Seddigh, J. Solano and I. Stojmenovic, RNG and internal node based broadcasting in one-to-one wireless networks, ACM Mob. Comput. Commun. Rev. 5 (2001) 37-44.

[14] H. Takagi and L. Kleinrock, Optimal transmission ranges for randomly distributed packet radio terminals, IEEE Trans. Commun. 32(3) (1984) 246-257.

[15] G. Toussaint, The relative neighborhood graph of a finite planar set, Pattern Recogn. 12 (1980) 261-268.

[16] P.-J. Wan and C.-W. Yi, On the longest edge of gabriel graphs in wireless ad hoc networks, IEEE Trans. Parall. Distri. Syst. 18 (2007) 111-125.

[17] P.-J. Wan, C.-W. Yi, F. Yao and X. Jia, Asymptotic critical transmission radius for greedy forward routing in wireless ad hoc networks, ACM MOBIHOC (2006), pp. 25-36. 
FA 1

March 26, 2009 20:14 WSPC/257-DMAA 00009 